\title{
Up front hepatectomy for metastatic rectal carcinoma - reversed, liver first approach. Early experience with 15 patients.
}

\author{
M. STRAKA ${ }^{1, *}$, M. SKROVINA ${ }^{1}$, R. SOUMAROVA ${ }^{2}$, R. KOTASEK ${ }^{2}$, L. BURDA ${ }^{1}$, C. VOJTEK ${ }^{3}$ \\ ${ }^{1}$ Department of Surgery, Comprehensive Cancer Centre and AGEL Research and Training Institute Novy Jicin, Hospital Novy Jicin, Czech Repub- \\ lic; ${ }^{2}$ Department of Oncology and Radiotherapy, Comprehensive Cancer Centre Centre and AGEL Research and Training Institute Novy Jicin, \\ Hospital Novy Jicin, Czech Republic; ${ }^{3}$ Department of Radiology, Comprehensive Cancer Centre and AGEL Research and Training Institute Novy \\ Jicin, Hospital Novy Jicin, Czech Republic
}

*Correspondence: martin.straka@nemnj.cz

Received October 9, 2013 / Accepted November 28, 2013

\begin{abstract}
Timing and sequence of therapeutic interventions in patients with colorectal cancer (CRC) and synchronous liver metastases is a matter of ongoing discussion. The aim of this report is to show the feasibility and safety of a reversed strategy in patients with up front resectable synchronous liver metastases.

Consecutive series of 15 patients with locally advanced rectal carcinoma and liver synchronous metastases where up front liver resection was carried out as an initial intervention is presented. Local treatment of both, metastatic disease and primary tumor, was preferred. Liver resection was followed by neoadjuvant (preoperative) concomitant radiochemotherapy (RCT) for local pelvic disease control and subsequent resection of rectum. Systemic adjuvant chemotherapy was placed at the end of the entire treatment cycle.

All 15 patients after up front hepatectomy were able to proceed with their treatment plan. 14 patients completed their RCT for primary tumor and subsequent rectal resection was successfully caried out in 12 of them. 3 patients showed complete clinical response on cross sectional imaging and a careful „wait-and-see“ policy was adopted for them. In two patients metastatic disease progression was noticed during the treatment cycle.

Liver first approach in patients with up front resectable colorectal liver metastases (CRLM) is safe and feasible. Local neoadjuvant treatment after CRLM resection may result in preoperative downsizing or even complete clinical response of the primary tumor. Reversed strategy may to a degree eliminate negative oncologic impact of surgical complications after rectal surgery as CRLM has been already addressed.
\end{abstract}

Key words: hepatectomy, liver first approach, reversed strategy, synchronous liver metastases

Completing the whole therapeutic plan in patients with stage IV. rectal cancer is of utmost importance in order to achieve long term survival. The liver first approach avoids possible liver metastases progression into an inoperable state while operating on primary [1-3]. Moreover, anastomotic complication after rectal resection in classical, „rectum first“ approach may lead to a delay of systemic treatment, again with a possible conversion of initially resectable liver disease into an advanced inoperable state [2,4]. A generally lower hepatectomy complication rate [5-10] compared to complication rate after rectal resection, seems to apply to the liver first approach as well ( $27.3 \%$ vs $44.4 \%$ reported by de Jong et al. in the liver first approach) [4]. The reversed strategy therefore, seems to give a better opportunity for the patient to get addressed both, liver disease and primary [11]. The proportion of patients who complete their full treatment protocol after liver first approach is reported about 73-81 $\%$ by several authors $[4,6,12,13]$. In 2012 analysis based on LiverMetSurvey by Andres et al., up to $80 \%$ of patients resected liver first successfully underwent the complete treatment plan, compared to less than $30 \%$ of those undergoing the classical rectum first approach [14].

Preoperative, neoadjuvant concomitant radiochemotherapy (RCT) results in better local control in patients with advanced rectal cancer $[15,16,17]$. In patients presenting with metastatic disease, initial systemic chemotherapy is preferred $[11,18]$. Radiotherapy in this setting is usually administered in adjuvant fashion after rectal resection to ensure local control. Patients 
experiencing serious anastomotic complications after rectal resection are often left without radiotherapy at all. Reversed approach in this particular group of patients addresses liver disease early and enables them to benefit from neodjuvant RCT for rectal tumor. Systemic chemotherapy may then follow surgery in an adjuvant fashion. Risk of local complications from a tumor left in situ while resecting liver disease and receiving RCT is relatively low $[1,19,22,23]$ and can be avoided by constructing loop ileostomy during the hepatic procedure if the patient is already symptomatic.

\section{Patients and methods}

During the period of 2011-2012, 75 hepatectomies were performed at our institution. In 56 cases, surgery was indicated for CRLM, 35 from these for synchronous lesions. Based on a multidisciplinary cancer team conference, there were 15 consecutive patients with locally advanced rectal carcinoma (T3b and more, or $\mathrm{N}+$ ) presenting with synchronous up front resectable, liver only metastastases. The staging assessment of the primary tumor was based on colonoscopy, histology, computed tomography (CT), magnetic resonance imaging (MRI) and transrectal ultrasonography. CRLM were assessed as clearly resectable by liver surgeon. The staging was based on standard 3 phase CT scanning, or MRI. Positron emission tomography (PET) was not considered mandatory. After the panel discussion, every patient was given an informed consent and agreed to the proposed treatment strategy. This group was retrospectively compared to a corresponding cohort of patients treated in a period of 2009-2010 when classical, rectum first approach was used.

\section{Results}

1-7 CRLM lesions per patient were resected during up front hepatectomy taking into account a general tendency towards liver sparing surgery. Despite prevailing non anatomic, limited resections, 6 major procedures (right, left hemihepatectomy with or without concomitant contralateral metastasectomy) had to be performed. Altogether, 6 major hepatic resections, 2 bisegmentectomies and 23 complete metastasectomies were performed for 46 lesions in 15 patients (Table 1). The loop ileostomy in 6 patients and terminal sigmoideostomy in one case were constructed at the time of hepatectomy for symptomatic rectal tumor obstruction. The aim was to relieve present obstruction symptoms which would be further worsened by upcoming RCT and to protect subsequent low colorectal anastomosis after the planned rectal resection.

No patient experienced primary tumor related complications (obstruction, bleeding) during hepatectomy and neoadjuvant treatment period. None of the patients within the group died and no postoperative liver failure, bleeding or biliary leak was noticed. No patient required hepatectomy related reoperation. One liver resection surface infection was noticed and this was evaluated as an ascendent nosocomial infection through an intraoperatively placed drain. This patient had prolonged hospital stay and percutaneous intervention was needed to drain residual perihepatic collection. The same patient experienced subsequent complications after both rectal resection and resection of protective loop ileostomy. In the end, an immune system disorder was revealed and immunomodulation treatment was installed accordingly. In another patient a twist of loop ileostomy occurred, requiring a simple orthotopic reinsertion during the same hospitalization. This did not result in any delay in his treatment protocol. We assigned this complication to surgeon's technical failure.

After liver first resection all but one patient completed their neoadjuvant treatment for better local control of the primary tumor. Patient number 9 (Table 1) had been treated for Hodgkin disease in the past, in which he received radiotherapy (40 Gy). This was the reason why he was not eligible for concomitant RCT after liver first hepatectomy. Because of this modified treatment plan patient number 9 was excluded from further consideration. Neoadjuvant RCT resulted in downsizing of the primary tumor (RECIST criteria used) or in downstaging of the $\mathrm{N}$ or $\mathrm{T}$ status in 10 patients (Table 1). In 2 patients stable disease after RCT was suggested on CT/MRI and was subsequently confirmed on their histopathology. The other 2 patients were evaluated as having complete clinical response based on MRI finding. In these patients a cautious „wait-and-see“ policy was adopted with repeated sigmoideoscopy and multiple biopsies every 3 months.

In the classical, „rectum first approach period“ (2009-2010) there were 16 patients with clearly resectable synchronous CRLM and locally advanced rectal cancer identified retrospectively. After rectal resection only 12 of them went on with their treatment plan and had hepatectomy for CRLM. 4 patients ( $25 \%$ ) had no hepatectomy because either the disease progression or complication after rectal surgery. This is in contrast to „liver first approach period“ (2011-2012) where all 15 liver first resected patients either underwent rectal resection, or had complete clinical response after RCT. 13 liver first resected patients have their „surgical cycle“ completed so far (hepatectomy followed by RCT and subsequent resection of rectum). 2 patients do not have their rectal tumor resected yet, both are experiencing complete clinical response with close follow up. Disease progression during the treatment cycle occurred in two patients. In one patient second line systemic chemotherapy resulted in complete clinical response both within the liver and the pelvis, another patient was given chemotherapy with palliative intent .

An average interval between hepatectomy and neoadjuvant treatment was 28,79 (14-55) days and average „delay“ of rectal resection after liver first plus neoadjuvant RCT was 128,77 (91260 ) days. Adjuvant chemotherapy (6 months after colorectal surgery) with disease restaging is not finished yet and further evaluation is ahead. 
Table 1. Patient characteristics with regard to type of surgical intervention, effect of preoperative RCT and intervals between hepatectomy and neoadjuvant RCT, hepatectomy and resection of the primary and hepatectomy and adjuvant chemotherapy.

\begin{tabular}{|c|c|c|c|c|c|c|}
\hline & Type of hepatectomy & $\begin{array}{l}\text { Resection of } \\
\text { rectum }\end{array}$ & RCT effect on primary & $\begin{array}{c}\text { Hepatectomy- } \\
\text { RCT (days) }\end{array}$ & $\begin{array}{l}\text { Hepatectomy- } \\
\text { colectomy (days) }\end{array}$ & $\begin{array}{l}\text { Hepatectomy- adjuvant } \\
\text { chemotherapy (days) }\end{array}$ \\
\hline & $2 \mathrm{x} C M$ & LAR & PR/N downstage/histopathol. + & 14 & 100 & 141 \\
\hline & biseg S2+3, $2 \times$ CM, PIS & LAR & PR/N downstage/histopathol. + & 29 & 118 & refused by patient \\
\hline 3. & biseg S2+3, 3 x CM, CHE, PIS & LAR & PR/N downstage/histopathol. - & 19 & 91 & 152 \\
\hline & CM, CHE, PIS ${ }^{* 1 p s k}$ & LAR & PR/N downstage/histopathol. + & 24 & 113 & 141 \\
\hline & LHH S1-4, PIS & LAR & PR/N downstage/histopathol.+ & 28 & 115 & 163 \\
\hline & $2 \times$ CM, PIS & LAR & PR/N downstage/histopathol. + & 21 & 106 & 155 \\
\hline & $2 \mathrm{x}$ CM, sigmoideostomy & wait and see & $\mathrm{CR}$ & 55 & - & - \\
\hline & RHH S5-8 & APR & PR/N downstage/histopathol.+ & 30 & 119 & 143 \\
\hline 9. & RHH S5-8 & $\begin{array}{l}\text { wait and see } \\
\text { failure - LAR }\end{array}$ & $\mathrm{CR}+$ progression & - & 260 & 289 \\
\hline & RHH S5-8, CM & LAR & PR/CR/T , N downstage/histopathol. T+, N+ & 29 & 155 & 198 \\
\hline & $2 \times$ CM, CHCE, PIS & LAR & PR/T, N downstage/histopathol. T-, N+ & 47 & 131 & 168 \\
\hline 12. & LHH S2-4, 3 x CM & wait and see & $\mathrm{CR}$ & 35 & - & - \\
\hline & $3 \times \mathrm{CM}$ & $\mathrm{APR}+\mathrm{IORT}$ & PR/T , N downstage/histopathol. + & 16 & 108 & 147 \\
\hline & RHH S5-8 & LAR & PR/T, N downstage/histopathol. T+, N- & 27 & 126 & 161 \\
\hline & $2 \times \mathrm{CM}, \mathrm{CHCE}^{\star l p s k}$ & TPE & $\mathrm{SD}$ & 29 & 132 & 167 \\
\hline
\end{tabular}

Legend : patients are sorted in chronological order; type of hepatectomy : CM - complete metastasectomy, LHH - left hemihepatectomy, RHH - right hemihepatectomy, biseg - bisegmentectomy, CHE - cholecystectomy, ${ }^{\star l p s k}$ - laparoscopic resection; resection of rectum : LAR - low anterior resection, PIS - protective loop ileostomy, TPE - total pelvic exenteration, IORT - intraoperative radiotherapy, APR - abdominoperineal resection; RCT effect on primary : CR - complete clinical response, PR - partial response, SD - stable disease, (CR, PR, SD assesment on restaging imaging after neoadjuvant treatment according to RECIST criteria), TNM downstaging based on cross sectional imaging after neoadjuvant treatment, histopathol. + suggested downstaging confirmed on final histopathology, histopathol. - suggested downstaging not confirmed on final histopathology for T or N status respectively

\section{Discussion}

In the past, a presence of colorectal cancer metastases within the liver was considered contraindication to curative intent surgery as disease dissemination was universally expected. Nowadays liver only, or liver limited metastatic disease is accepted to be localized, provided that overall dissemination of the disease cannot be proved. The fact, that the liver is the only site of CRLM in $30 \%$ of patients is widely acknowledged $[22,23]$. At present it seems, that we can in some cases even say, that patient has limited extrahepatic disease and this applies to peritoneum as well. Altough patients with extrahepatic metastatic involvement have a much worse prognosis, surgery is worthwhile in carefully selected cases and the line between local and systemic disease is becoming less clear [24-26]. In this study, based on this trend, a patient with synchronous liver only CRLM was considered to have a localized disease in two compartments. In our therapeutic protocol, emphasis was put on local treatment of liver metastases and local pelvic control by RCT followed by rectal resection. Prehepatectomy chemotherapy was not used and systemic adjuvant therapy was launched after complete surgical resection of the disease. Giving up prehepatectomy chemotherapy delays early treatment of micrometastatic disease, but the benefit of prehepatectomy chemotherapy in resectable CRLM is currently questioned. Even though it appears, that a short course of preoperative chemotherapy is common practice in a majority of centres
[6,27-29], some authors assume that the role of prehepatectomy chemotherapy should be reconsidered [8,30-36]. In a systematic review of Lehmann et al. [34] routine use of preoperative chemotherapy in resectable CRLM could not be recommended. To resect CRLM up front is an intriguing option for many surgeons. An intact liver without chemotherapy- induced liver injury means more predictable future for liver remnant (FLR) function, lower complication rate and no missing lesions issue [37-42]. On the other hand, the most important benefit of neoadjuvant preoperative chemotherapy is probably to identify and select patients with favourable tumor biology [43]. When resecting liver first without preoperative chemotherapy, patients run the risk of subsequent tumor non response and rapid disease progression. Non response to systemic therapy is currently attributed to particularly aggressive tumor biology. Consensus on management of such aggressive tumors is not settled yet, because non response or disease progression while on certain chemotherapy regimen does not necessarilly mean non response at all. Interestingly, proceeding to liver resection, if possible, is also considered a valuable option even in this subgroup of patients [31,32,34]. Preoperative use of chemotherapy offers an early treatment of micrometastatic disease and a possibility of tumor shrinkage enabling for liver sparing surgery in some cases. However, missing lesions issue and liver toxicity are the most important drawbacks of this strategy [27,41,44,45-48]. Pros and cons of preoperative chemotherapy make it hard to identify 
which therapeutic sequence is the best in synchronous metastatic CRC patients [2,30,49]. Disregarding the initial strategy, achieving radical tumor clearance must be the goal, as this is the only chance for survival.

There are several reports on nonoperative, „wait-andsee" strategy for patients with complete clinical response of primary rectal tumor after neoadjuvant RCT. It seems, that about $80 \%$ of carefully selected patients with complete clinical response can be safely spared rectal resection and reach long term survival with rectum left in situ $[50,51,52]$. Intensive follow-up is mandatory. In our series, there were 3 patients with complete clinical response after neoadjuvant treatment based on MRI, repeated sigmoideoscopy and multiple biopsies every 3 months. „Wait-and-see“ policy failed however in one patient (patient number 9 (Table 1)). Eventually, this patient had his rectal resection 260 days after hepatectomy with a final pathological staging equivalent to inicial pretreatment evaluation. The other two patients remain without any evidence of primary tumor recurrence.

According to a recent systemic review in as many as $19 \%$ of patients disease progression may occur during the liver first protocol period [53]. In our series, two patients experienced metastatic disease progression during a treatment cycle. 5 year overall survival from $31 \%$ to $41 \%$ with recurrence rate from $22 \%$ to $70 \%$ can be expected for reversed strategy according to lately published report [54]. For patients from this series the short follow-up period currently does not allow for recurrence or overall survival data evaluation.

\section{Conclusion}

Liver first strategy compared with classical, rectum first approach, enables to accomplish the whole treatment plan to a higher proportion of patients presenting with metastatic rectal carcinoma. Addressing liver disease early avoids liver progression into an inoperable state while resecting primary. Moreover, the reversed strategy partially eliminates negative oncologic impact of surgical complications after rectal surgery, as CRLM have been already addressed. After up front resection of CRLM, patients with locally advanced rectal carcinoma can benefit from concomitant RCT leading to better local pelvic disease control, possible preoperative downsizing, or even complete clinical response of primary tumor. The benefits of liver first approach are, however dependent on low hepatectomy complication rate, which has to be kept to minimum in order to prevent a delay in planned treatment protocol.

\section{References}

[1] GRUNDMANN RT. Current state of surgical treatment of liver metastases from colorectal cancer. World J Gastrointest Surg 2011; 3: 183-196. http://dx.doi.org/10.4240/wjgs.v3.i12.183

[2] TSOULFAS G, PRAMATEFTAKIS MG. Management of rectal cancer and liver metastatic disease: which comes first? Int J
SurgOncol 2012; 2012: 196908. http://dx.doi.org/10.1155/ $\underline{2012 / 196908}$

[3] MENTHA G, MAJNO P, TERRAZ S, RUBBIA-BRANDT L, GERVAZ $P$ et al. Treatment strategies for the management of advanced colorectal liver metastases detected synchronously with the primary tumor. Eur J Surg Oncol 2007; 33(Suppl. 2): 76-83. http://dx.doi.org/10.1016/j.ejso.2007.09.016

[4] DE JONG MC, VAN DAM RM, MAAS M, BEMELMANS MHA, OLDE DAMINK SWM et al. The liver-first approach for synchronous colorectal liver metastasis: a 5-year singlecentre experience. HPB 2011; 13: 745-752. http://dx.doi. org/10.1111/j.1477-2574.2011.00372.x

[5] FAHY BN, FISHER CP. Synchronous resection of colorectal primary and hepatic metastasis. J Gastrointest Oncol 2012; 3: $48-58$.

[6] LAM VWT, LAURENCE JM, PANG T, JOHNSTON E, HOLLANDS MJ et al. A systematic review of a liver-first approach in patients with colorectal cancer and synchronous colorectal liver metastases. $\mathrm{HPB}(2013)$. [Epub ahead of print] http:// dx.doi.org/10.1111/hpb.12083

[7] MIRNEZAMI R, MIRNEZAMI AH, CHANDRAKUMARAN K, HILAL MA, PEARCE NW et al. Short- and long-term outcomes after laparoscopic and open hepatic resection: systematic review and meta-analysis. HPB (Oxford) 2011; 13: 295-308. http://dx.doi.org/10.1111/j.1477-2574.2011.00295. $\underline{\mathrm{x}}$

[8] NEUMANN UP, SEEHOFER D, NEUHAUS P. The surgical treatment of hepatic metastases in colorectal carcinoma. Dtsch Arztebl Int 2010; 107: 335-342.

[9] NYGARD IE, LASSEN K, KJÆVE J, REVHAUG A. Mortality and survival rates after elective hepatic surgery in a low-volume centre are comparable to those of high-volume centres. ISRN Surg 2012; 2012: 783932. http://dx.doi.org/10.5402/ 2012/783932

[10] REDDY SK, BARBAS AS, TURLEY RS, STEEL JL, TSUNG A et al. A standard definition of major hepatectomy: resection of four or more liver segments. HPB (Oxford) 2011; 13: 494-502. http://dx.doi.org/10.1111/j.1477-2574.2011.00330. $\underline{\mathrm{x}}$

[11] VAN DESSEL E, FIERENS K, PATTYN P, VAN NIEUWENHOVE Y, BERREVOET F, et al. Defining the optimal therapy sequence in synchronous resectable liver metastases from colorectal cancer: a decision analysis approach. Acta Chir Belg 2009; 109: 317-320.

[12] VERHOEF C, VAN DER POOL AE, NUYTTENS JJ, PLANTING AS, EGGERMONT AM et al. The „liver-first approach“ for patients with locally advanced rectal cancer and synchronous liver metastases. Dis Colon Rectum 2009; 52: 23-30. http://dx.doi.org/10.1007/DCR.0b013e318197939a

[13] MENTHA G, MAJNO PE, ANDRES A, RUBBIA-BRANDT L, MOREL $P$ et al. Neoadjuvant chemotherapy and resection of advanced synchronous liver metastases before treatment of the colorectal primary. Br J Surg 2006; 93: 872-878. http://dx.doi. org/10.1002/bjs.5346

[14] ANDRES A, TOSO C, ADAM R, BARROSO E, HUBERT C et al. A survival analysis of the liver-first reversed management of advanced simultaneous colorectal liver metastases: a Liver- 
MetSurvey-based study. Ann Surg 2012; 256: 772-8; discussion 778-9. http://dx.doi.org/10.1097/SLA.0b013e3182734423

[15] GARAJOVA I, DI GIROLAMO S, DE ROSA F, CORBELLI J, AGOSTINI V et al. Neoadjuvant Treatment in Rectal Cancer: Actual Status. Chemother Res Pract 2011; 2011: 839742. http://dx.doi.org/10.1155/2011/839742

[16] SAUER R, FIETKAU R, WITTEKIND C, MARTUS P, RODEL $\mathrm{C}$ et al. Adjuvant versus neodajuvant radiochemotherapy for locally advanced rectal cancer. Strahlenther Onkol 2001; 177: 173-181. http://dx.doi.org/10.1007/PL00002396

[17] SIEGEL R, BUROCK S, WERNECKE KD, KRETZSCHMAR $A$, DIETEL $M$ et al. Preoperative short-course radiotherapy versus combined radiochemotherapy in locally advanced rectal cancer: a multi-centre prospectively randomised study of the Berlin Cancer Society. BMC Cancer 2009; 9: 50. http:// dx.doi.org/10.1186/1471-2407-9-50

[18] MENTHA G, ROTH AD, TERRAZ S, GIOSTRA E, GERVAZ $P$ et al. ,Liver first' approach in the treatment of colorectal cancer with synchronous liver metastases. Dig Surg 2008; 25: 430-435. http://dx.doi.org/10.1159/000184734

[19] HOPT UT, DROGNITZ O, NEEFF H. [Timing of resection in patients with colorectal carcinoma and synchronous liver metastases][Article in German]. Zentralbl Chir 2009; 134: 425-9. http://dx.doi.org/10.1055/s-0029-1224612

[20] POULTSIDES GA, SERVAIS EL, SALTZ LB, PATIL S, KEMENY NE et al. Outcome of primary tumor in patients with synchronous stage IV colorectal cancer receiving combination chemotherapy without surgery as initial treatment. J Clin Oncol 2009; 27: 3379-84. http://dx.doi.org/10.1200/ LCO.2008.20.9817

[21] SCHEER MG, SLOOTS CE, VAN DER WILT GJ, RUERS TJ. Management of patients with asymptomatic colorectal cancer and synchronous irresectable metastases. Ann Oncol 2008; 19: 1829-1835. http://dx.doi.org/10.1093/annonc/ $\underline{\operatorname{mdn} 398}$

[22] JONSSON K, GRONDAHL G, SALO M, TINGSTEDT B, ANDERSSON R. Repeated Liver Resection for Colorectal Liver Metastases: A Comparison with Primary Liver Resections concerning Perioperative and Long-Term Outcome. Gastroenterol Res Pract. 2012; 2012: 568214. http://dx.doi. org/10.1155/2012/568214

[23] WEISS MJ, D'ANGELICA MI. Patient selection for hepatic resection for metastatic colorectal cancer. J Gastrointest Oncol 2012; 3: 3-10.

[24] CARPIZO DR, ARE C, JARNAGIN W, DEMATTEO R, FONG $Y$ et al. Liver resection for metastatic colorectal cancer in patients with concurrent extrahepatic disease: results in 127 patients treated at a single center. Ann Surg Oncol 2009; 16: 2138-2146. http://dx.doi.org/10.1245/s10434-009-0521-6

[25] CARPIZO DR, D'ANGELICA M. Liver resection for metastatic colorectal cancer in the presence of extrahepatic disease. Lancet Oncol 2009; 10: 801-809. http://dx.doi.org/10.1016/ $\underline{\text { S1470-2045(09)70081-6 }}$

[26] ELIAS D, OUELLET JF, BELLON N, PIGNON JP, POCARD $\mathrm{M}$ et al. Extrahepatic disease does not contraindicate hepatectomy for colorectal liver metastases. Br J Surg 2003; 90 : 567-574. http://dx.doi.org/10.1002/bjs.4071
[27] BENOIST S, NORDLINGER B. The role of preoperative chemotherapy in patients with resectable colorectal liver metastases. Ann Surg Oncol 2009; 16: 2385-2390. http://dx.doi. org/10.1245/s10434-009-0492-7

[28] NORDLINGER B, SORBYE H, GLIMELIUS B, POSTON GJ, SCHLAG PM et al. Perioperative chemotherapy with FOLFOX4 and surgery versus surgery alone for resectable liver metastases from colorectal cancer (EORTC Intergroup trial 40983): a randomised controlled trial. The Lancet 2008; 371 : 1007-1016. http://dx.doi.org/10.1016/S0140-6736(08)60455-9

[29] WIESER M, SAUERLAND S, ARNOLD D, SCHMIEGEL W, REINACHER-SCHICK A. Peri-operative chemotherapy for the treatment of resectable liver metastases from colorectal cancer: A systematic review and meta-analysis of randomized trials. BMC Cancer 2010; 10: 309. http://dx.doi.org/10.1186/ 1471-2407-10-309

[30] ADAM R, BHANGUI P, POSTON G, MIRZA D, NUZZO G et al. Is perioperative chemotherapy useful for solitary, metachronous, colorectal liver metastases? Ann Surg 2010; 252: 774-787. http://dx.doi.org/10.1097/SLA.0b013e3181fcf3e3

[31] CAI GX, CAI SJ. Multi-modality treatment of colorectal liver metastases. World J Gastroenterol 2012; 18: 16-24. http:// dx.doi.org/10.3748/wig.v18.i1.16

[32] GALLAGHER DJ, ZHENG J, CAPANU M, HAVILAND D, PATY P et al. Response to neoadjuvant chemotherapy does not predict overall survival for patients with synchronous colorectal hepatic metastases. Ann Surg Oncol 2009; 16: 1844-1851. http://dx.doi.org/10.1245/s10434-009-0348-1

[33] HADDAD AJ, BANI HANI M, PAWLIK TM, CUNNINGHAM SC. Colorectal liver metastases. Int J Surg Oncol 2011; 2011: 285840. http://dx.doi.org/10.1155/2011/285840

[34] LEHMANN K, RICKENBACHER A, WEBER A, PESTALOZZI BC, CLAVIEN PA. Chemotherapy before liver resection of colorectal metastases: friend or foe? Ann Surg 2012; 255: 237-247. http://dx.doi.org/10.1097/SLA.0b013e3182356236

[35] NANJI S, CLEARY S, RYAN P, GUINDI M, SELVARAJAH S et al. Up-front hepatic resection for metastatic colorectal cancer results in favorable long-term survival. Ann Surg Oncol 2013; 20: 295-304. http://dx.doi.org/10.1245/s10434-012-2424-1

[36] SHAH K, CLARY B. Synchronous colorectal liver metastases: The surgeon as oncologist" perspective. Minerva Chir 2013; 68: 49-76.

[37] ALOIA T, SEBAGH M, PLASSE M, KARAM V, LEVI F et al. Liver histology and surgical outcomes after preoperative chemotherapy with fluorouracil plus oxaliplatin in colorectal cancer liver metastases. J Clin Oncol 2006; 24: 4983-90. http://dx.doi.org/10.1200/JCO.2006.05.8156

[38] DIMITROULIS D, NIKITEASN, TROUPIS T, PATSOURAS D, SKANDALAKIS P et al. Role of surgery in colorectal liver metastases: Too early or too late? World J Gastroenterol 2010; 16: 3484-3490. http://dx.doi.org/10.3748/wjg.v16.i28.3484

[39] KISHI Y, ZORZI D, CONTRERAS CM, MARU DM, KOPETZ $S$ et al. Extended preoperative chemotherapy does not improve pathologic response and increases postoperative liver insufficiency after hepatic resection for colorectal liver metastases. Ann Surg Oncol 2010; 17: 2870-6. http://dx.doi. org/10.1245/s10434-010-1166-1 
[40] NAKANO H, OUSSOULTZOGLOU E, ROSSO E, CASNEDI S, CHENARD-NEU MP et al. Sinusoidal injury increases morbidity after major hepatectomy in patients with colorectal liver metastases receiving preoperative chemotherapy. Ann Surg 2008; 247: 118-24. http://dx.doi. org/10.1097/SLA.0b013e31815774de

[41] ROBINSON SM, WILSON CH, BURT AD, MANAS DM, WHITE SA. Chemotherapy-Associated Liver Injury in Patients with Colorectal Liver Metastases: A Systematic Review and Meta-analysis. Ann Surg Oncol 2012; 19: 4287-99. http://dx.doi.org/10.1245/s10434-012-2438-8

[42] VAUTHEYJN, PAWLIK TM, RIBERO D, WU TT, ZORZI D et al. Chemotherapy regimen predicts steatohepatitis and an increase in 90-day mortality after surgery for hepatic colorectal metastases. J Clin Oncol 2006; 24: 2065-72. http://dx.doi. org/10.1200/JCO.2005.05.3074

[43] ADAM R, PASCAL G, CASTAING D, AZOULAY D, DELVART $V$ et al. Tumor progression while on chemotherapy: a contraindication to liver resection for multiple colorectal metastases? Ann Surg 2004; 240: 1052-61; discussion 1061-4. http://dx.doi.org/10.1097/01.sla.0000145964.08365.01

[44] REDDY SK, BARBAS AS, CLARY BM. Synchronous colorectal liver metastases: is it time to reconsider traditional paradigms of management? Ann Surg Oncol 2009; 16: 2395-410. http:// dx.doi.org/10.1245/s10434-009-0372-1

[45] KHAN AZ, MORRIS-STIFF G, MAKUUCHI M. Patterns of chemotherapy-induced hepatic injury and their implications for patients undergoing liver resection for colorectal liver metastases. J Hepatobiliary Pancreat Surg 2009; 16: 137-144. http://dx.doi.org/10.1007/s00534-008-0016-Z

[46] SOUBRANE O, BROUQUET A, ZALINSKI S, TERRIS B, BREZAULT $C$ et al. Predicting high grade lesions of sinusoidal obstruction syndrome related to oxaliplatin-based chemotherapy for colorectal liver metastases: correlation with post-hepatectomy outcome. Ann Surg 2010; 251: 454-460. http://dx.doi.org/10.1097/SLA.0b013e3181c79403

[47] TAMANDL D, KLINGER M, EIPELDAUER S, HERBERGER B, KACZIREK K et al. Sinusoidal obstruction syndrome im- pairs long-term outcome of colorectal liver metastases treated with resection after neoadjuvant chemotherapy. Ann Surg Oncol 2011; 18: 421-430. http://dx.doi.org/10.1245/s10434$\underline{010-1317-4}$

[48] ZORZI D, LAURENT A, PAWLIK TM, LAUWERS GY, VAUTHEY JN et al. Chemotherapy-associated hepatotoxicity and surgery for colorectal liver metastases. Br J Surg 2007; 94 : 274-286. http://dx.doi.org/10.1002/bjs.5719

[49] REDDY SK, ZORZI D, LUM YW, BARBAS AS, PAWLIK TM et al. Timing of multimodality therapy for resectable synchronous colorectal liver metastases: a retrospective multiinstitutional analysis. Ann Surg Oncol 2009; 16: 1809-19. http://dx.doi.org/10.1245/s10434-008-0181-y

[50] MAAS M, BEETS-TAN RG, LAMBREGTS DM, LAMMERING G, NELEMANS PJ et al. Wait-and-see policy for clinical complete responders after chemoradiation for rectal cancer. J Clin Oncol 2011; 29: 4633-40. http://dx.doi.org/10.1200/ LCO.2011.37.7176

[51] SMiTH JD, RUBY JA, GOODMAN KA, SALTZ LB, GULLIEM JG et al. Nonoperative management of rectal cancer with complete clinical response after neoadjuvant therapy. Ann Surg 2012; 256: 965-72. http://dx.doi.org/ 10.1097/SLA.0b013e3182759f1c

[52] HABR-GAMA A, PEREZ RO, NADALIN W, SABBAGA J, RIBEIRO U Jr et al. Operative versus nonoperative treatment for stage 0 distal rectal cancer following chemoradiation therapy: long-term results. Ann Surg 2004; 240: 711-7; discussion 717-8.

[53] JEGATHEESWARAN S, MASON JM, HANCOCK HC, SIRIWARDENA AK. The liver-first approach to the management of colorectal cancer with synchronous hepatic metastases: a systemic review. JAMA Surg 2013; 148: 385-91. http:// dx.doi.org/10.1001/jamasurg.2013.1216

[54] DE ROSA A, GOMEZ D, BROOKS A, CAMERON IC. Liverfirst" approach for synchronous colorectal liver metastases: is this a justafiable approach? J Hepatobiliary Pancreat Sci 2013; 20: 263-70. http://dx.doi.org/10.1007/s00534-012$\underline{0583-\mathrm{x}}$ 